\title{
REVIEW
}

\section{Progress in Clinical Trials of Tumor Immunodrugs in the United States}

\author{
Li Wan, Ye Zhang* \\ The First Affiliated Hospital of Bengbu Medical College, Bengbu 233004, Anhui, China
}

Corresponding Author: Ye Zhang, the First Affiliated Hospital of Bengbu Medical College, 287 Changhuai Road, Bengbu 233004, Anhui, China. E-mail: 1wan19@126.com

\begin{abstract}
Malignant tumors are a key factor that threatens human health. With the continuous development of molecular biology, immunology and oncology, tumor immunodrugs have gradually become an effective means to suppress malignant tumors after surgery, radiotherapy and chemotherapy. The United States, as a leading country in the development of tumor immunodrugs, has rich clinical trial practice experience and comprehensive clinical trial considerations. Therefore, this article intends to explore the progress of clinical trials of tumor immunodrugs in the United States in terms of the alternative endpoints of clinical trials, clinical trials of adolescent tumor patients, and the design of a single clinical trial main plan for multiple drugs and/or multiple tumor subgroups, thereby providing a reference for optimizing the design of clinical trials in China, expanding the application scope of tumor immunodrugs, and speeding up the process of marketing new drugs.

Keywords

Tumor immunity; clinical trials; alternative endpoint; adolescent patients with cancer; clinical trial design
\end{abstract}

\section{INTRODUCTION}

Malignant tumor is one of the important killers that threaten human health in the world, and it is also an important factor that seriously endanger human life and restrict social and economic development ${ }^{[1]}$. At present, traditional tumor treatment methods in the world mainly include surgery, radiotherapy and chemotherapy. However, there are many disadvantages and obvious adverse reactions in the traditional treatment. With the continuous development and cross-penetration of oncology, immunology, molecular biology and other related disciplines ${ }^{[2]}$, tumor immunotherapy has gradually developed and has become an important means to effectively overcome malignant tumors.

The United States is a country with rapid development of the tumor immune drug industry, and has been in the leading position in the field of tumor immunity worldwide. In order to improve the availability of patients' drugs, the U.S. Food and Drug Administration (FDA ) has accelerated the time to market for new oncology drugs from multiple perspectives, including setting up special review channels, simplifying the application process for marketing, and optimizing the design of clinical trials. Among them, clinical trials is the research phase with the largest investment of time and capital in the research and

(C) The Author(s) 2019. Open Access This article is under the terms of Creative Commons Attribution 4.0 International License (https://creativecommons.org/licenses/by/4.0/), which permits unrestricted use, sharing, adaptation, distribution and reproduction in any medium or format, as long as you give appropriate credit to the original author(s) and the source, provide a link to the Creative Commons license, and indicate if changes were made. 
development of new drugs. Good clinical trials can effectively shorten the time to market for drugs. Therefore, this article discusses the progress of clinical trials of tumor immunodrugs in the United States, with a view to optimizing the design of clinical trials of tumor immunodrugs in China and further accelerating the marketing process of tumor immunodrugs.

\section{DEVELOPMENT BACKGROUND OF TUMOR IMMUNODRUGS}

Tumor immunodrugs are drugs that can inhibit or kill tumor cells by mobilizing or stimulating the body's own immune function to enhance various anti-tumor capabilities in the microenvironment around the tumor. In the middle of the 19th century, German pathologist Virchow ${ }^{[1]}$ observed the appearance of immune infiltration in human tumor tissues, and proposed the concept of tumor immunity for the first time. Since then, tumor immunodrugs have developed rapidly and have attracted widespread attention within and outside the industry. At present, programmed death-1 (PD-1)/programmed death ligand 1 (PDL1) inhibitors and chimeric antibody receptor engineered T cell (CAR-T) therapy has become a hot topic at home and abroad.

The development of tumor immunodrugs in the United States has been at the forefront of the world; and for the clinical research in progress, the United States has always been in an unshakable position in the field of tumor immunity. Studies by Ting Zhang ${ }^{[3]}$ et al., show that in the countries/regions developing tumor immunotherapy drugs, the United States has 2,307 tumor immunotherapy drugs, accounting for $56.31 \%$ of the total global drugs; there are 690 tumor immunotherapy drugs in China, accounting for $16.84 \%$ of the total global drugs. Although China ranks second only to the United States in the world, there is still a large gap with the United States, and the number of drugs for clinical research is less than one third of that in the United States. Therefore, whether it is the number of approved drugs, the number of drugs under investigation, or the clinical research of tumor immunotherapy drugs, the United States has strong advantages and good practical experience. So it is of great significance to explore the relevant content of clinical trials of tumor immunodrugs in the United States to improve the level of clinical trials of tumor immunodrugs in China.

\section{PROGRESS IN CLINICAL TRIALS OF TUMOR IMMUNODRUGS IN THE UNITED STATES}

As a global leader in the pharmaceutical industry, the clinical trials of anti-tumor drugs in the United States are more innovative than those in other countries. Whether it is the clinical trial endpoint, the included population, or the clinical trial design, FDA has optimized and improved the existing clinical trial scheme from multiple perspectives to accelerate the marketing process of tumor immunodrugs to the greatest extent, and meet the drug demand of tumor patients.

\subsection{Endpoints of Clinical Trials of Tumor Immunodrugs}

\subsubsection{Legislation and Regulatory Requirements for the Effectiveness of New Drugs}

The U.S. Federal Food, Drug, and Cosmetic Act (FD\&C Act) states that applicants are required to provide substantial evidence from well-controlled clinical studies to prove the effectiveness of the drug. The accelerated approval regulations (21 CFR 314, subpart H and 21 CFR 60,1 subpart E) provide that the FDA can accelerated the approval of the drug or biological product designed to treat serious or life-threatening disease that can significantly improve current therapies or provide previously untreated therapies, based on alternative endpoints that can reasonably predict clinical benefit. Thus, the alternative endpoints have become an effective basis for accelerating the market for new drugs.

\subsubsection{Endpoints of Traditional Support for Tumor Immunodrug Reviews}

For the standard approval pathway, survival improvement is the primary indicator for predicting clinical benefit. However, the FDA approved other alternative endpoints for drug marketing, including overall survival, endpoints based on tumor evaluation [such as disease-free survival (DFS)/event-free survival (DES), objective response rate (ORR), time to progression (TP), progression-free survival (PFS), etc.], and endpoints based on symptom evaluation (patient-reported outcome) ${ }^{[4]}$. For accelerated approval, alternative endpoints must be able to reasonably predict clinical benefit. Among them, ORR is the most commonly used alternative endpoint to support accelerated approval. Because ORR can be directly attributed to drug effects, when no single-arm trial is available for patients with refractory tumors, ORR can be selected as a clinical alternative endpoint. Whether to use endpoints based on tumor evaluation like ORR or PFS, as the indicator of accelerated or standard approval will depend on the background of the disease, the degree of impact of the disease, and other influencing factors.

Based on specific application conditions, the clinical endpoints of different tumors can be used for different purposes 
(i.e., clinical endpoints that support the clinical benefit of the standard approval pathway, alternative endpoints that support the standard approval pathway, and alternative endpoints that support the accelerated approval pathway). The clinical endpoint selected for different clinical trials should be different according to the specific disease. Factors such as the degree of the efficacy, duration of efficacy, response intensity, available therapies, tumor location, clinical outcomes of delayed disease progression and risk-benefit relationships should all be taken into account. The endpoints of each approval route are compared in Table $1^{[5]}$.

Table 1 Comparison of approved endpoints of main tumor immunodrugs

\begin{tabular}{|c|c|c|c|c|c|c|}
\hline \multirow[b]{2}{*}{ Endpoints } & \multicolumn{3}{|c|}{ Types of Endpoints } & \multicolumn{3}{|c|}{ Suggestions for Clinical Trial Design } \\
\hline & Clinical Endpoints & $\begin{array}{c}\text { Alternative } \\
\text { Endpoints for } \\
\text { Standard Approval }\end{array}$ & $\begin{array}{c}\text { Alternative Endpoints } \\
\text { for Accelerated } \\
\text { Approval }\end{array}$ & $\begin{array}{c}\text { Randomized } \\
\text { Trial }\end{array}$ & $\begin{array}{l}\text { Single-arm } \\
\text { Test }\end{array}$ & $\begin{array}{c}\text { Independent Blind } \\
\text { Evaluation }\end{array}$ \\
\hline Overall Survival (OS) & $\sqrt{ }$ & & & $\sqrt{ }$ & & \\
\hline $\begin{array}{l}\text { Symptom Endpoint (report } \\
\text { patient's results) }\end{array}$ & $\sqrt{ }$ & & & $\sqrt{ }$ & & \\
\hline DFS/DES & $\sqrt{ }$ & $\sqrt{ }$ & $\sqrt{ }$ & $\sqrt{ }$ & & $\sqrt{ } a^{a}$ \\
\hline ORR & $\sqrt{ }$ & $\sqrt{ }$ & $\sqrt{ }$ & $\sqrt{ }$ & $\sqrt{ }$ & $\sqrt{ }$ \\
\hline & $\sqrt{ }$ & $\sqrt{ }$ & $\sqrt{ }$ & $\sqrt{ }$ & $\sqrt{ }$ & $\sqrt{ }$ \\
\hline $\mathrm{PFS} / \mathrm{TP}$ & $\sqrt{ }$ & $\sqrt{ }$ & $\sqrt{ }$ & $\sqrt{ }$ & & $\sqrt{ } \mathrm{a}^{\mathrm{a}}$ \\
\hline
\end{tabular}

a: Not often recommended; DFS: disease-free survival; DES: event-free survival; ORR: objective response rate; CR: complete remission; PFS: progression-free survival; TP: time to progression

\subsubsection{Emerging Clinical Alternative Endpoints}

In addition to the above alternative endpoints, with the advancement of science, the research and development of tumor products has become more and more in-depth. Many emerging alternative endpoints that can be used to support the approval of tumor products have gradually been discovered.

Minimal residual disease (MRD) is an indicator for measuring cancer cells remaining in the body of patients with hematological malignancies after a long period of treatment or after achieving complete remission or complete response (CR), which is the main reason for the recurrence of hematological tumors. MRD is also a general measurement index for detecting tumor burden, and it has a variety of potential regulatory and clinical application values as a biomarker. Depending on the clinical situation, MRD can reflect the patient's response to treatment and can also be used as a prognostic tool to assess the risk of future recurrence in patients. At the same time, MRD can also be used to enrich clinical trial populations or as a basis for guiding populations to be assigned to specific treatment departments in clinical trials ${ }^{[6]}$. Based on the above, MRD can be used as an alternative endpoint for accelerated approval of acute lymphoblastic leukemia.

Non-metastatic castration-resistant prostate cancer (nmCRPC) is a kind of malignant tumor with decreased testosterone levels but still increased prostate-specific antigen (PSA) and no imaging evidence of distant metastases. Although early detection of localized prostate cancer can get the curative effect through surgery or radiotherapy to certain extent; after receiving local treatment of recurrent prostate cancer (such as surgery, radiotherapy) and subsequent androgen deprivation therapy (ADT), PSA will continue to increase in many patients. Because the treatment time of patients with nmCRPC may be longer and patients are prone to distant tumor metastasis or death due to PSA elevation, OS cannot be used as the main endpoint to support the approval of products for the treatment of this disease. Based on the above considerations, the U.S. Oncologic Drugs Advisory Committee (ODAC) believes that within an acceptable safety range, metastatic-free survival (MFS) can be regarded as the clinical benefit endpoint for early treatment of nmCRPC ${ }^{[7]}$.

In addition, serum asparaginase can be used as a standard route to approve alternative endpoints for acute lymphoblastic leukemia; immune-related response criteria (irRC), such as irORR, irDCR, and irPFS, can also provide more trial alternative endpoints options for tumor immunotherapy ${ }^{[8]}$. However, due to the low amount of data available in clinical trials, it may not be sufficient to prove that new alternative endpoints are validated based on reasonable and sufficient data. Therefore, the FDA recommends that sponsors should strengthen data collection capabilities in post-marketing studies to identify possible 
alternative endpoints ${ }^{[9]}$.

If the clinical trial sponsor wants to use the emerging alternative endpoint in the clinical development plan, the FDA recommends that before starting the clinical trial, the sponsor should fully discuss this section with the corresponding department or office of FDA to determine whether the endpoint can reasonably predict the clinical benefits.

\subsection{Inclusion of Adolescents (12-17 years) in Clinical Trials of Cancer in Adults}

Currently, the legal minimum age to participate in adult clinical trials is generally 18 years old ${ }^{[10]}$. But it is also because of age that adolescents are usually not eligible to participate in adult tumor clinical trials, and many drugs are usually not tested in pediatric clinical trials until adult drug approval. As a result, adolescents have much later access to potentially effective treatments than adults. In addition, it may be difficult to include them in pediatric clinical trials due to the off-label medication of adolescents. Studies have shown that Gustave Roussy Research Center conducted solid tumor or lymphoma phase I and phase II trials during 2012 to 2017, and only 65 of the 465 open trials included adolescents aged 12-17. In other words, patients in this age group account for less than $15 \%$ of trials in the early stage ${ }^{[11]}$. Therefore, the treatment of adolescent cancer patients is facing great challenges. To address these issues, the FDA considers including adolescents in clinical trials of adult tumors with appropriate diseases and targets, so as to obtain drugs for adolescent cancer patients as early as possible ${ }^{[12]}$.

\subsubsection{Inclusion Criteria for Adolescents}

FDA believes that if the histology and biologic behavior for the same symptoms in adult and adolescent patients are the same during drug development, or if their drug molecular targets are related, then adolescents can be included in adult tumor clinical trials. Therefore, the FDA has made relevant recommendations based on the different stages of drug development. For the first human trial or dose escalation trial, sponsors can register eligible adolescent patients after obtaining early adult pharmacokinetic and toxicological data. Prior to this, sponsors should consult with the FDA's review department to determine the amount and type of adult data needed before registering adolescent patients. If adolescent patients are required to participate in an early dose group trial, the sponsor should ensure that the doses they take meet the requirements of 21 CFR 50.52 . For activity estimating or confirmatory trials, FDA recommends that adolescent patients can register at the same time as adult patients.

\subsubsection{Dosage Recommendations}

Based on the influence of body size on pharmacokinetics, general exposure and drug clearance in adolescents and adults are often very similar. Therefore, the dosage selection for adolescent patients should be the same as that of adults, and the dose should be adjusted according to body size (weight or surface area) or take a fixed dose (that is, the dose is not adjusted based on weight or surface area). The specific recommended dose should be determined by the pharmacokinetic characteristics of the study drug, taking into account the influence of body size on pharmacokinetics, the therapeutic index of the drug, and dose \& exposure-response relationships. Pharmacokinetic samples from adolescent patients should be collected and analyzed when they are included in drug development programs to prove that the drug system exposure of adolescent and adult patients is similar.

The FDA believes that the dosage for adolescents can be deduced based on the dosage for adults. First of all, for a drug that is adjusted based on the weight of an adult, the teen should be consistent with the adjusted dose of an adult of the same weight $\left(\mathrm{mg} \bullet \mathrm{kg}^{-1} / \mathrm{mg}^{-2} \mathrm{~m}^{-2}\right.$. For drugs taken at a fixed dose, a minimum weight threshold should be defined to prevent adolescents who weigh less than average from exceeding adult exposure. According to the FDA's adult pharmacokinetic analysis of oncology drugs, $40 \mathrm{~kg}$ (12-year-old average weight) is usually the lower limit of the weight range, but this lower limit may be change according to the characteristics of the drug (the influence of body size on pharmacokinetics, the therapeutic index, and dose $\&$ exposure-response relationships). In general, adolescents weighing more than $40 \mathrm{~kg}$ can receive the same fixed dose as adults. For adolescents weighing less than $40 \mathrm{~kg}$, the adjusted dose should be taken based on body weight $\left(\mathrm{mg}^{\circ} \mathrm{kg}^{-1}\right)$ or body surface area $\left(\mathrm{mg} \bullet \mathrm{m}^{-2}\right)$. The adjusted dose should refer to the physical parameters of an adult (for example, the average or median weight of an adult of $70 \mathrm{~kg}$ or the body surface area of an adult patient based on available data).

\subsubsection{Safety Monitoring}

FDA believes that the safety data collected during the trial should be closely investigated for age-related differences. During early trials, indicators of developmental toxicity (e.g., growth disorders, fertility problems) that require long-term follow-up may not be evaluated. However, where feasible, sponsors should develop a plan for longitudinal evaluation that can 
assess potential developmental toxicity, especially in early clinical trials. Unless clinical and/or non-clinical data do not provide sufficient toxicity data, juvenile animal studies are usually not required until adolescents are enrolled in a clinical trial of tumors $^{[13]}$.

Although the FDA currently does not issue regulations or complete clinical guidelines for the inclusion of adolescents in adult tumor clinical trials (only draft guidelines are currently issued). However, in actual clinical practice, some pharmaceutical companies have included adolescent patients into clinical trials. For example, on August 30, 2017, the FDA approved the first CAR-T oncology immune drug, tisagenlecleucel (brand name: Kymriah), in a clinical study B2202, which set up a dedicated pediatric and adolescent patient study group; and the age subgroups are 2 to 11 years old (50\%), 12 to 17 years old (25\%) and $\geqslant 17$ years old $(25 \%)$. Also, no differences in effectiveness or safety were observed between different age subgroups or compared with the young adults group in the trial.

\subsection{Design a Single Clinical Trial Master Protocols for Multiple Drugs and/or Multiple Tumor Subgroups}

With the advent of the era of "personalized medicine" and "precision medicine", the rapid development of genomics and molecular biology has a significant impact on clinical trials of tumor drugs. The classification of tumor is no longer limited to histopathological classification, but also began to focus on the genetic or molecular changes that cause tumorigenesis. At the same time, in the treatment of tumors, people have also begun to shift from traditional treatment models for tumor tissue sites to the treatment model for tumor specific genetic variation ${ }^{[14]}$. Therefore, the development of efficient new clinical trial designs has become a hot topic within and outside the industry.

In 2014, the American Association for Cancer Research (AACR) pointed out that master protocols of innovative clinical trials for precision oncology medicine will become the mainstream of future anti-tumor drug clinical trial design.

\subsubsection{Definition and Type of Master Protocol}

A master protocol is defined as a trial protocol with multiple subgroups and different goals that require coordinated efforts of multiple parties to evaluate one or more disease subtypes / one or more study drugs throughout the trial ${ }^{[15]}$. There are two main types, namely basket trials and umbrella trials.

A basket trial is a master protocol designed to test a single study drug or combination of drugs in different groups defined by disease stage, histology, number of previous therapies, genetic or other biomarkers or demographic characteristics (see Figure 1). The subgroups in basket trials are usually designed as single-arm activity-estimating trials with the overall response rate (ORR) as the primary endpoint. When strong response signals are observed in a substudy, the substudy can be extended to obtain data to support marketing approval. Each sub-study should include specific goals, scientific basis for inclusion in each population, and detailed statistical analysis plan (SAP), including the reasonableness of the sample size and invalid stopping rules.

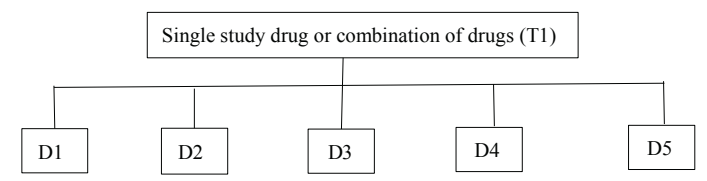

T: study drug; D: definition of subgroups in multiple disease subtypes

Figure 1 Schematic diagram of the master protocol of basket trial design

Umbrella trials are the master protocols designed to evaluate multiple study drugs administered as a single drug or combination of drugs in a single disease population (see Figure 2). Sub-studies in an umbrella trial may include dose-finding components to determine the safe dose of the study drug combination before an activity-estimating component is performed.

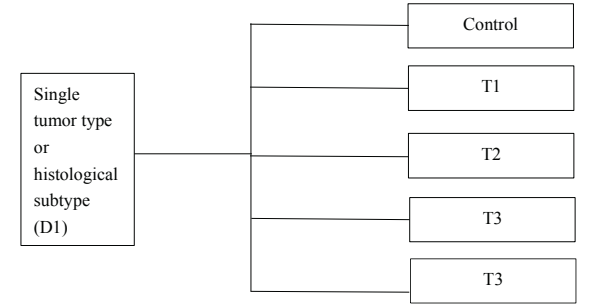

T: study drug; D: definition of subgroups in a single disease subtypes; TX: future treatment group

Figure 2 Schematic diagram of the master protocol of umbrella trial design 


\subsubsection{Comparison of the Master Protocol Trial Design with Previous Clinical Trial Design}

Compared with the previous clinical trial design, because of the smaller sample size and shorter research and observation time, the master protocol design can effectively shorten the clinical trial process and accelerate the public access to safe and effective drugs and drug treatment combinations. Second, the master plan design can combine effective methods such as sharing control arms and/or using a centralized data acquisition system to improve efficiency. At the same time, the inclusion of a large number of potential marker treatment combinations in the same study can enhance the motivation of research stakeholders, including treating physicians, participating institutions, applicants and patients (see Table 2). Therefore, the master protocol has made great contributions to the accelerated development of precision treatment drugs and the development of clinical oncology. However, there may be some disadvantages in the implementation and analysis of the master protocol. The biggest problem is security monitoring. For example, when multiple drugs are in a multi-arms trial and there is a lack of a single internal control over these drugs, if an adverse event occurs, the investigator may not be able to determine which drug caused the adverse event. On the other hand, because many drugs are studies across multiple protocols and investigational new drug applications (INDs), it is difficult to assess the safety of any given study drug. As people's understanding of the mutation spectrum in tumors is not comprehensive, there are still many gene mutations being researched and developed; and some "driver mutations" may become "passenger genes" in a few years ${ }^{[16]}$. Therefore, whether it is a basket test or an umbrella test, it still needs further refinement and research in order to provide subjects with more optimized and accurate clinical trials.

Table 2 Comparison of the master protocol trial design with traditional clinical trial design

\begin{tabular}{|c|c|c|c|}
\hline Types & Traditional clinical trial design & \multicolumn{2}{|c|}{ Master protocol trial design } \\
\hline Methods & $\begin{array}{l}\text { cohort study, randomized } \\
\text { controlled study, etc. }\end{array}$ & basket trial & umbrella trial \\
\hline Objectives & $\begin{array}{l}\text { a single targeted therapy for a } \\
\text { single disease }\end{array}$ & $\begin{array}{l}\text { single targeted therapy in the context of } \\
\text { multiple diseases or disease subtypes }\end{array}$ & $\begin{array}{l}\text { multiple targeted therapies in the context of } \\
\text { a single disease subtype }\end{array}$ \\
\hline Advantages & $\begin{array}{l}\text { the test scheme is relatively } \\
\text { simple and highly feasible }\end{array}$ & $\begin{array}{l}\text { molecular targeted drugs can be provided } \\
\text { for patients with the same cancerous gene } \\
\text { type; this trial design can reduce the time } \\
\text { for initial diagnosis and/or qualification } \\
\text { confirmation and subsequent cohort allocation } \\
\text { and treatment initiation; cohort sample sizes } \\
\text { are small, and single-stage or dual-stage trial } \\
\text { design is often used; if the design is good, the } \\
\text { corresponding results can be obtained quickly }\end{array}$ & $\begin{array}{l}\text { tumor heterogeneity is less likely to occur } \\
\text { in a given trial group }{ }^{[17]} \text {; when randomized } \\
\text { targeted therapies and non-targeted } \\
\text { therapies are performed in the cohort, } \\
\text { especially when the marker is negative, } \\
\text { the drug's mechanism can be evaluated } \\
\text { more comprehensively; and prognostic } \\
\text { versus predictive marker effects can be } \\
\text { distinguished empirically }\end{array}$ \\
\hline Disadvantages & $\begin{array}{l}\text { the choice of subjects is narrow; } \\
\text { time and capital consumption } \\
\text { are large; different dosage } \\
\text { regimens may produce different } \\
\text { dose-limiting toxicity and } \\
\text { maximum tolerated dose }\end{array}$ & $\begin{array}{l}\text { the environment and location of tumor } \\
\text { development may affect tumor mutation } \\
\text { spectrum, and may lead to different predicted } \\
\text { treatment responses across similar spectrum }\end{array}$ & $\begin{array}{l}\text { for rare tumor diseases, if it is necessary } \\
\text { to further subdivide the subtypes of tumor } \\
\text { types that have been identified as rare } \\
\text { diseases by changing the molecule, it may } \\
\text { lead to poor accumulation in the population } \\
\text { and even slow the overall trial }{ }^{[17]}\end{array}$ \\
\hline
\end{tabular}

\subsubsection{Application of Master Plan Design in Tumor Immunodrugs}

On May 23, 2017, FDA accelerated the approval of Merck's PD-1 inhibitor pembrolizumab (brand name: Keytruda) for the treatment of adult and pediatric cancer patients with microsatellite instability-high (MSI-H) or defective mismatch repair (dMMR), and unresectable or metastatic solid tumors ${ }^{[18]}$. This is the first tumor immunotherapy approved by the US FDA that is not related to the origin of tumor but differentiated according to the same pathological gene. This means that no matter what kind of tumor, as long as it contains MSI-H/dMMR features, it can be treated by using Keytruda. 149 patients with MSI-H or dMMMR tumors covered 15 types of cancers, including colorectal cancer, endometrial cancer, gastric cancer, and breast cancer. The clinical study used ORR as the endpoint, with an ORR of 39.6\% (95\% CI: 31.7, 47.9). Patients who respond to pembrolizumab have a sustained response time of 6 months or more ${ }^{[19]}$. In addition to pembrolizumab, on July 10, 2018, the 
U.S. FDA also accelerated the approval of ipilimumab in combination with nivolumab for the treatment of MSI-H or dMMR metastatic colorectal cancer (mCRC) aged 12 years and older.

Microsatellite instability (MSI) refers to the change in microsatellite length caused by the insertion or deletion of a simple repeat sequence caused by a replication error of a microsatellite in tumor tissue compared to normal tissue, which shows the change of structural allele size, while MSI-H is the most unstable one ${ }^{[20]}$. Mimatch repair (MMR) is an important mechanism for maintaining the stability of chromosomes in cells. If MMR-related genes are mutated, MSI-H will occur during the DNA replication process, so it will not be repaired in time. This is one of the reasons for tumorigenesis . Because MSI-H and dMMR are two common genetic abnormalities, tumors with these abnormalities are widely distributed and can appear in many parts of the colorectum, endometrium, gastrointestinal tract, breast, prostate, bladder, and thyroid gland ( (see Table 3$)^{[21]}$. Therefore, the concept of guiding different tumor treatments based on the same biomolecular characteristics rather than the origin of tumor will bring a new and open therapeutic thinking to tumor treatment, which is also an important step in the practice of precision medicine ${ }^{[22]}$.

Table 3 Proportion of MSI-H / dMMMR-positive patients

\begin{tabular}{cc}
\hline Types of Cancer & Proportion (\%) \\
\hline Thyroid cancer & 63 \\
Dermolipoma & $35 \sim 60$ \\
Endometrial cancer & $22 \sim 33$ \\
Gastric cancer & 22 \\
Liver cancer & 16 \\
Colon cancer & 13 \\
Melanoma & 11 \\
\hline
\end{tabular}

\section{PROBLEMS AND PROSPECTS}

Clinical trials are not only the driving force behind the development of medicine, but also a key stage in accelerating the process of new drugs marketing and promoting the innovation and development of the pharmaceutical industry. As the most innovative product of anti-tumor drugs, tumor immunodrugs need better clinical trial design to identify the effectiveness and safety of drugs and expand the clinical application of drugs. However, there are still many problems and challenges in the clinical trials of tumor immunodrugs in China. (1) generally, ORR is selected as the index of effectiveness of phase I clinical trials of tumor immune drugs in China, PFS is selected for phase II clinical trials, and OS is selected for phase III clinical trials. This method can make the entire clinical research cycle relatively short ${ }^{[23]}$; but for some malignant tumors with special pathological mechanisms, this approach may cause different evaluation results of clinical endpoints. (2) With the increasing incidence of malignant tumors in China, clinical trials targeting adults (18 to 70 years old) and children ( 0 to 14 years old) have been the research mainstream in the field of medicine. However, the adolescent group is often ignored by people. Some adolescent patients may not be able to participate in clinical trials and not be able to obtain the latest treatments ${ }^{\text {[24] }}$, which ultimately leads to a decline in the quality of life and even death of patients. (3) Due to the historical reason that the Chinese pharmaceutical industry is dominated by generic drugs, some sponsors and researchers have limited clinical trial design capabilities, especially inadequate innovative trial design ${ }^{[25]}$ (such as adaptive research, basket research, umbrella research, etc.). Most clinical trial designs still refer to foreign studies and lack consideration of domestic influencing factors.

Based on the above problems, in order to meet the unmet clinical needs of patients, improve the availability of medications for patients, and ensure the safety of medications for patients, the author proposes the following suggestions for reference. (1) the clinical alternative endpoint is an important indicator to measure the clinical benefits of tumor immunodrugs. Clinical trial researchers should use the big data analysis platform to explore potential effective alternative endpoints, and strengthen postmarketing research and data collection capabilities to provide a reasonable data basis for potential alternative endpoints, so as to improve the prediction ability and accuracy of clinical benefits. (2) adolescents are often neglected in clinical research in China. This is also the main reason for the lower cure rate of adolescent patients than adults and children. Therefore, China's 
regulatory authorities may consider that for the first human trial or dose escalation test, the eligible young patients can be included to expand the application of tumor drugs and obtain drugs for such groups as soon as possible, according to different tumor types (bone, sarcoma, etc.), after obtaining early adult pharmacokinetic data and toxicological data. At the same time, as adolescents are still at the critical period of body and organ development, special consideration should be given to the design of clinical trials for them. It is recommended that China's regulatory authorities should organize relevant experts to formulate corresponding clinical trial guidelines to provide key references for enterprises and researchers. (3) the design of clinical trials is the key to clinical research. Excellent and reasonable trial schemes can effectively achieve the expected clinical goals. Relevant researchers in China should start from the source and refer to foreign advanced clinical trial design concepts (basket trial, umbrella trial, etc.) in combination with China's actual conditions, and explore more innovative and cutting-edge design schemes, optimize the clinical trial management system, and further promote the top-level design and capacity building by using advanced technology \& real-world data, and provides the source power for the development of tumor immunodrug industry in China.

\section{REFERENCE}

[1] Han YM, Cao XT. Grasp the trends of tumor immunotherapy accurately to promote its orderly and healthy development in China. Chin J Cancer Biother 2017,24(1):2-5.

[2] Lu ZH, Shen L. Current strategies and prospects of cancer immunotherapy. Chin J Frontiers Med Sci (Electronic Version) 2017,9(10):1-7,225.

[3] Zhang T, Ouyang ZL. The development situation analysis of tumor immunotherapy drugs based on Cortellis database. Chin J New Drugs 2019,28(1):1-9.

[4] Sun YX, Wei FF, Yang Y. Development and Application of Surrogate Endpoints in FDA's Drug Review Process. Chin Pharm J 2017,52(5):414-419.

[5] FDA. Clinical Trial Endpoints for the Approval of Cancer Drugs and Biologics Guidance for Industry. [2018-12-19]. https://www.fda.gov/downloads/Drugs/GuidanceComplianceRegulatoryInformation/Guidances/UCM071590.pdf.

[6] FDA. Hematologic malignancies: regulatory considerations for use of minimal residual disease in development of drug and biological products for treatment. [2018-10-05]. https://www.fda.gov/downloads/Drugs/GuidanceComplianceRegulatoryIn formation/Guidances/UCM623333.pdf.

[7] FDA. Nonmetastatic, Castration Resistant Prostate Cancer: Considerations for Metastasis-Free Survival Endpoint in Clinical Trials. [2018-11-1]. https://www.fda.gov/downloads/Drugs/GuidanceComplianceRegulatoryInformation/ Guidances/UCM625703.pdf.

[8] Zhao H, Ren XB. Key points and difficulties in clinical trials of cellular immunotherapy of cancer. Chin J Cancer Biother 2016,23(6):741-744.

[9] FDA. What is the challenge of using novel biomarkers as reasonably likely surrogate endpoints in medical productdevelopment? https:/www.fda.gov/Drugs/DevelopmentApprovalProcess/DevelopmentResources/ucm606684.htm.

[10] Archie B, Troy B, Michael M. Adolescents and young adults with cancer. Cancer 2006,107(S7):1645-1655.

[11] Xiao Q. Health: Barriers to early clinical trial access for adolescents and young people persist [2018-10-22]. https:// baijiahao.baidu.com/s?id=1615001140025565118\&wfr=spider \& for $=$ pc.

[12] FDA. Considerations for the inclusion of adolescent patients in adult oncology clinical trials guidance for industry draft guidance. [2018-06-01]. https://www.fda.gov/downloads/Drugs/GuidanceComplianceRegulatoryInformation/Guidances/ UCM609513.pdf.

[13] Leighton JK, Saber H, Reaman G, Pazdur R. An FDA oncology view of juvenile animal studies in support of initial pediatric trials for anticancer drugs. Regul Toxicol Pharmacol, 2016,79:142-143.

[14] Hu HX, Wang L, Li CJ, Xia JL. Application of basket test in the research and development of anti-tumor targeted drugs. Chin J Health Statistics 2018,35(3):477-480.

[15] FDA. Master protocols: efficient clinical trial design strategies to expedite development of oncology drugs and biologics 
guidance for industry. [2018-09-28]. https://www.fda.gov/downloads/Drugs/GuidanceComplianceRegulatoryInformation/ Guidances/UCM621817.pdf.

[16] MedSci. Innovative clinical trials of precision cancer medicine: umbrella trial and basket trial. [2015-12-14]. http://www. medsci.cn/article /show_article.do?id=2bb7605e $3 \mathrm{db}$.

[17] Renfro LA, Sargent DJ. Statistical controversies in clinical research: basket trials, umbrella trials, and other master protocols: a review and examples. Ann Oncol 2017,1(28):34-43.

[18] FDA. FDA grants accelerated approval to pembrolizumab for first tissue/site agnostic indication. [2017-05-23]. https:// www.fda.gov/Drugs/InformationOnDrugs/ApprovedDrugs/ucm560040.htm.

[19] FDA. FDA grants accelerated approval to pembrolizumab for firsttissue/site agnostic indication.[2017-05-23]. https://www. fda.gov/Drugs/InformationOnDrugs/ApprovedDrugs/ucm560040.htm.

[20] Peng JL, Tang T, Ye ZL, Shao Q, Huang LY, Deng L, Wang F, Shao JY. The relationship of microsatellite instability state with loss of mismatch repair proteins and clinical pathological characteristics in sporadic colorectal cancers. Chin J Cancer Biother 2015,22(4):479-483.

[21] Yao HM. FDA approved the world's first "broad-spectrum" anticancer drug: cancer treatment into a new era. [2017-5-27]. http://www.istis.sh.cn/list /list.aspx?id=10657.

[22] Yuce Biology. How to consider oncology guided by biomarker? [2017-05-25]. https://www.sohu.com/a/143466299_730586.

[23] Wang MX, Lou DH. Consideration of General Design for Clinical Trials of Innovative Antitumor Biotechnology Drugs. Chinese Journal of Rational Drug Use 2015,12(10):51-56.

[24] Dingxiangyuan. Survey of Cancer Status in Adolescents and Youth. [2016-04-14]. http://pediatr.dxy.cn/article/ 488001 trace $=$ drugs.

[25] CAEFI, Clinical evaluation and Research Committee of Chinese Pharmaceutical Association, Center for regulatory science excellence of APEC, Peking University Clinical Research Institute, China Pharmaceutical Enterprises Association, China Pharmaceutical Industry Association, China Chamber of Commerce for Import\&Export of Medicines \& Health Products. Current difficulties and challenges during the design and conduct stages of clinical trials in China. Chin J New Drugs 2018,27(11):1225-232. 